\title{
Reflets
}

Revue d'intervention sociale et communautaire

\section{Inégalités et politiques alternatives}

Entrevue avec David Macdonald

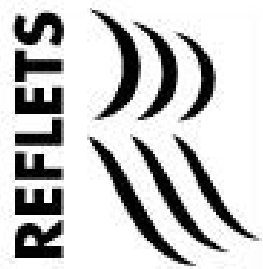

\section{Komi Hemedzo}

Volume 24, numéro 1, printemps 2018

URI : https://id.erudit.org/iderudit/1051518ar

DOI : https://doi.org/10.7202/1051518ar

Aller au sommaire du numéro

Éditeur(s)

Reflets, Revue d'intervention sociale et communautaire

ISSN

1203-4576 (imprimé)

1712-8498 (numérique)

Découvrir la revue

Citer ce document

Hemedzo, K. (2018). Inégalités et politiques alternatives : entrevue avec David Macdonald. Reflets, 24(1), 18-24. https://doi.org/10.7202/1051518ar d'utilisation que vous pouvez consulter en ligne.

https://apropos.erudit.org/fr/usagers/politique-dutilisation/ 


\section{Inégalités et politiques alternatives}

Entrevue avec David Macdonald

Centre canadien de politiques alternatives (CCPA)

\section{Présentation de la personne et de son organisme}

David Macdonald est économiste principal au Centre canadien de politiques alternatives (CCPA). Le CCPA est un institut de recherche indépendant et non partisan qui sintéresse aux questions de justice sociale, économique et environnementale. Fondé en 1980, le CCPA est l'une des principales voix progressistes du Canada dans les débats sur les politiques publiques. Il est situé au 251, rue Bank, bureau 500, Ottawa, ON, Canada, K2P 1 X3.

Cette entrevue a été réalisée le 4 juillet 2017 par Komi Hemedzo et rédigée par Komi Hemedzo.

Reflets : Bonjour, Monsieur Macdonald. Pourriez-vous nous présenter le Centre canadien de politiques alternatives? Votre politique? Vos objectifs? Vos actions?

D. Macdonald: Nous avons plusieurs bureaux. Nous avons un bureau ici, à Ottawa, où nous examinons des enjeux nationaux et fédéraux. Nous examinons l'inégalité des revenus nationaux et des politiques du gouvernement fédéral de manière progressiste. Nous avons aussi des bureaux provinciaux. Cependant, nous n'avons pas de bureau dans toutes les provinces. Nous avons un bureau ou un organisme dans les provinces avec lesquelles nous travaillons. Ces bureaux examinent des inégalités de revenus, des politiques nationales, provinciales et fédérales. Ils travaillent sur les politiques provinciales ou municipales.

Souvent, ces bureaux travaillent majoritairement sur les politiques provinciales comme notre bureau en ColombieBritannique, qui examine les changements climatiques. À propos 
de tous ces sujets, nous écrivons des rapports et nous examinons des tendances que nous voyons dans l'économie et dans la politique économique fédérale et provinciale. Nous publions ces rapports. C'est aux représentants des médias et du gouvernement d'examiner ce que nous écrivons. Notre but est de produire de bonnes analyses sur une base empirique que d'autres institutions peuvent utiliser pour faire de bonnes critiques politiques.

Nous sommes progressistes, nous ne sommes pas conservateurs. Quand nous examinons les inégalités, c'est qu'il y a quelque chose qui nous inquiète. Nous nous inquiétons donc des inégalités des revenus et des richesses. Nous nous inquiétons des niveaux de pauvreté, là où nous trouvons que le taux de pauvreté est élevé. Lorsque le taux de pauvreté et le taux des inégalités sont aussi élevés, cela nous inquiète.

Nos actions concrètes : c'est que nous publions des rapports et nous écrivons des blogues. Par exemple, nous faisons des analyses, mais nous sommes un organisme charitable, caritatif. Cependant, nous ne pouvons pas dire : «Appelle ton représentant ou ta représentante au Parlement et dis-lui de voter sur cette loici ou de voter contre cette loi-là. " Tout ce que nous pouvons faire, c'est de canaliser la politique du gouvernement et de dire quelles sont ses implications.

\section{Inégalités au Canada}

Reflets: Comment se présentent les inégalités au Canada? Quelles sont les différentes formes d'inégalités qu'on peut observer aujourd'hui au Canada?

D. Macdonald : Nous y avons travaillé beaucoup pendant ces dix dernières années dans le cadre de notre rapport sur les inégalités. Nous avons travaillé sur un de nos grands projets qui visent à analyser les inégalités et à examiner la tranche la plus riche de la population, c'est-à-dire les tranches de $1 \%$ ou de $10 \%$ de la population les plus riches en contraste avec le reste de la population, c'est-à-dire les plus pauvres et la classe moyenne. La tendance qu'on voit 
ici au Canada, et qu'il faut condamner et combattre, est que les plus riches voient leurs salaires augmenter de milliers de dollars chaque année par opposition aux pauvres. C'est quelque chose que nous examinons. Nous avons ainsi examiné les inégalités des revenus. Plus récemment, nous avons étudié l'inégalité des richesses par exemple à travers la situation des personnes qui ont accumulé des richesses notamment par le truchement des marchés immobiliers et des actions. Nous avons examiné aussi ce qui se passe du point de vue des inégalités de revenus par rapport aux identités. Par exemple, nous nous sommes interrogés sur comment se manifestent les inégalités chez les Autochtones. C'est quoi la pauvreté chez les Autochtones? Qu'est-ce qu'on voit si on examine l'inégalité sous l'angle des identités de sexe? L'identité par exemple des personnes qui sont immigrantes? Quelles sont leurs expériences? Alors, on constate que la pauvreté et les inégalités sont à un niveau très élevé. Mais on constate aussi des perspectives sur ces tendances.

\section{Le portrait de la situation aujourd'hui des inégalités au Canada et en Ontario}

Reflets: Quel est le portrait de la situation aujourd'hui des inégalités au Canada et en Ontario, si possible?

D. Macdonald : Nous avons un rapport que nous allons publier dans quelques semaines. Je ne connais pas actuellement le niveau de pauvreté en Ontario, mais ce qui va être intéressant à voir, c'est l'augmentation du salaire minimum qu'on va avoir en janvier de l'année prochaine. Et, dans deux ans, on va avoir une autre augmentation allant jusqu'à 15 dollars. Ce serait intéressant de voir l'augmentation des salaires des pauvres qui travaillent et d'analyser le niveau de pauvreté de ceux qui ne peuvent pas travailler. Ce serait alors intéressant de voir les inégalités des revenus, de voir l'écart avec les plus pauvres. La majorité de l'augmentation de salaire va bien sûr augmenter le pouvoir d'achat des plus pauvres. Mais, nous allons observer aussi la 
classe moyenne. Elle gagne plus que le salaire minimum. Va-telle avoir des augmentations de salaires à cause d'autres choses? C'est beaucoup moins certain.

Reflets: Comment expliquer, selon vous, ces réalités?

D. Macdonald : Cela dépend! C'est une question d'identité. Prenons par exemple les Autochtones. Le simple fait d'habiter dans les réserves les exclut de nos statistiques de pauvreté et d'inégalités.

Reflets : Pourquoi?

D. Macdonald : C'est par définition! Les autorités ont établi des définitions d'une manière à exclure les réserves. Dans un de nos projets, nous avons pris des lignes de définitions de pauvreté pour les appliquer aux réserves et aux Autochtones. Nous avons trouvé des taux qui sont très... très hauts.

Bien sûr, il y a des raisons historiques. Ce sont des endroits qui sont des juridictions du gouvernement fédéral. Et pendant les vingt dernières années, les gouvernants n'ont pas investi d'argent comme les gouvernements provinciaux ont investi dans les communautés qui sont proches des réserves. Pour cette raison, on voit des communautés autochtones moins développées que des communautés voisines dans les provinces.

Si l'on analyse les inégalités liées aux sexes, on constate aussi que les inégalités des sexes sont liées aux revenus. On note que l'histoire est unique au Canada. On a vu que les femmes ont augmenté leur taux d'emploi beaucoup plus rapidement que dans d'autres économies développées et qu'en même temps, c'est l'histoire canadienne. Les revenus des familles ont vite augmenté parce que les femmes travaillent par rapport aux années 19601970. Bien sûr qu'il y a divers types d'inégalités. Quand on va à un niveau plus bas, on constate que les identités donnent le sens à l'histoire et elles forment ces inégalités-là. Cela devient plus intéressant pour nous. 


\section{Reflets: Qu'est-ce qui explique ces inégalités avec les migrants?}

D. Macdonald : Avec les immigrants, c'est quelque chose qu'on n'a pas beaucoup examiné à cause du manque de données là-dessus. Ce n'est pas au même niveau qu'on les trouve pour les Canadiens non migrants. On trouve par exemple que les immigrants et les Autochtones qui ne vivent pas dans les réserves ont des taux de pauvreté assez similaires. C'est probablement à cause du fait qu'il est plus difficile de trouver un emploi qui a un salaire assez élevé. Cependant, les défis des immigrants sont différents de ceux des Autochtones. Chez les immigrants, on trouve que les niveaux d'éducation sont beaucoup plus élevés par rapport aux Autochtones, mais, au même moment, ils ne peuvent pas trouver de l'emploi et gagner de l'argent. Chez les Autochtones, on trouve le taux d'éducation plus bas. Paradoxalement, on trouve le même niveau de pauvreté que chez les migrants. Ce sont tous des exclus, car ils ne peuvent pas accéder au marché du travail pour des raisons différentes.

Reflets: Quelles sont les politiques publiques dans ce domaine?

D. Macdonald : On a utilisé les politiques de ces dix dernières années pour se rendre compte que l'inégalité des revenus est une chose dont il faut s'inquiéter en tant que pays. Comment est-ce qu'on peut combattre cette tendance dans l'inégalité des revenus? Bien sûr, dans les dernières années, on observe les systèmes de transfert et d'accession d'impôts puisque c'est ce que les gouvernements ont utilisé. Par exemple, le gouvernement fédéral a une nouvelle règle sur les taxes personnelles pour pouvoir en effet réduire les inégalités des revenus après les taxations. Ils ont utilisé cet argent-là pour augmenter les transferts aux familles avec enfants par l'entremise du programme. Ces décisions du gouvernement permettent de faire des transferts vers les familles. Je pense que les autres réponses sont possibles au moyen de la syndicalisation par exemple, mais on n'en est pas là. La question est vraiment : "Comment augmenter le salaire de la classe moyenne au même moment où l'on voit que les plus riches gagnent cinq ou dix 
fois plus? Et comment peut-on réduire cet écart? » L'une des manières, c'est d'avoir des augmentations du salaire pour la classe moyenne.

\section{Reflets : Leurs succès?}

D. Macdonald : Les transferts étaient assez populaires. Taxer les plus nantis, c'est quelque chose qui est populaire. Et l'introduction de nouvelles taxations pour les plus riches et au niveau des provinces était populaire. C'est quelque chose qui est accepté. L'un des rôles acceptables de nos gouvernements, c'est d'augmenter les taxes et de transférer cet argent aux moins nantis.

Reflets : Et leurs limites?

D. Macdonald : Chaque année, nous avons un grand projet que je gère et que j'appelle " notre budget automatique " au niveau du gouvernement fédéral. Chaque année, nous avons un chapitre qui examine les différents sujets ou politiques où l'on peut avoir des politiques progressistes. Il y a beaucoup de choses qu'on peut faire. On peut changer la politique de taxation pour que soient augmentées particulièrement les taxes sur le capital. Par exemple sur les stock options, on peut limiter les échappatoires. On peut gagner de l'argent de cette manière et on peut le dépenser de l'autre côté avec des transferts à la classe moyenne ou augmenter leur salaire. Cependant, il y a beaucoup plus de limites. Ces limites ne sont pas économiques. Ce sont des limites politiques.

\section{Ce que fait l'organisme comme politiques alternatives}

Reflets : Qu'est-ce que votre organisme fait comme politiques alternatives?

D. Macdonald : Comme je le disais, notre politique de budget alternatif est vraiment le plus grand livre concret que nous publions. Chaque année, nous publions un rapport en français et en anglais. Il est publié chaque année avant que le gouvernement ne présente son budget au printemps. Nous tenons compte de ce qui s'est produit l'année précédente. En plus, nous le publions 
dépendamment de ce qui s'est produit l'année précédente. Pendant les deux dernières années, nous avons constaté que nos recommandations ne sont pas complètement exécutées, mais que des portions ont été prises en compte dans le budget du gouvernement fédéral. Par contre, dans les budgets précédents, c'était beaucoup moins. On avait un gouvernement conservateur et non pas un gouvernement libéral. Comme actions concrètes, nous formulons des recommandations, et le gouvernement les applique en partie. Nous avons une influence sur les politiques.

Bien sûr, nous étions invités au conseil de finance, aux comités internationaux sur les changements climatiques, au comité sur l'environnement. Nos experts vont à ces comités tout le temps au niveau fédéral et au niveau provincial. Nous y participons pour donner nos opinions. Mais, notre publication est plus concrète, car les chapitres portent sur les étapes que nous examinons après pour analyser ce qui est inclus dans le budget du gouvernement.

Reflets: Merci pour cette entrevue.

D. Macdonald : Ça m’a fait plaisir. 